\title{
Reduction of myocardial reperfusion injury by aprotinin after regional ischemia and cardioplegic arrest
}

\author{
Tanveer A. Khan, $\mathrm{MD}^{\mathrm{a}}$ \\ Cesario Bianchi, MD, PhD \\ Pierre Voisine, $\mathrm{MD}^{\mathrm{a}}$ \\ Jun Feng, MD, PhD \\ Jeralyn Baker, CCPa \\ Melanie Hart, $\mathrm{PhD}^{\mathrm{b}}$ \\ Minoru Takahashi, $\mathrm{PhD}^{\mathrm{b}}$ \\ Greg Stahl, $\mathrm{PhD}^{\mathrm{b}}$ \\ Frank W. Sellke, MDa
}

From the Department of Surgery, Division of Cardiothoracic Surgery, Beth Israel Deaconess Medical Center and Harvard Medical School, Boston, Mass, ${ }^{\mathrm{a}}$ and the Department of Anesthesiology, Center for Experimental Therapeutics and Reperfusion Injury, Brigham and Women's Hospital and Harvard Medical School, Boston, Mass. $^{\text {b }}$

Funding was provided by National Institutes of Health grants R01 HL46716 (F.W.S.) and NRSA 1F32 HL69651 (T.A.K.) and by the Bayer Corporation.

Received for publication Oct 21, 2003; revisions requested Jan 27, 2004; revisions received Feb 9, 2004; accepted for publication March 17, 2004.

Address for reprints: Frank W. Sellke, MD, Division of Cardiothoracic Surgery, Beth Israel Deaconess Medical Center, 110 Francis St, LMOB 2A, Boston, MA 02215 (Email: fsellke@ caregroup.harvard.edu).

J Thorac Cardiovasc Surg 2004;128:602-8 $0022-5223 / \$ 30.00$

Copyright ( $\odot 2004$ by The American Association for Thoracic Surgery

doi:10.1016/j.jtcvs.2004.02.037
Background: Surgical coronary revascularization with cardiopulmonary bypass and cardioplegia has been associated with reperfusion injury. The serine protease inhibitor aprotinin has been suggested to reduce reperfusion injury, yet a clinically relevant study examining regional ischemia under conditions of cardiopulmonary bypass and cardioplegia has not been performed.

Methods: Pigs were subjected to 30 minutes of regional myocardial ischemia by distal left anterior descending coronary artery occlusion, followed by 60 minutes of cardiopulmonary bypass with 45 minutes of cardioplegic arrest and 90 minutes of post-cardiopulmonary bypass reperfusion. The treatment group $(\mathrm{n}=6)$ was administered aprotinin systemically (40,000 kallikrein-inhibiting units [KIU]/kg intravenous loading dose, 40,000 KIU/kg pump prime, and 10,000 KIU $\cdot \mathrm{kg}^{-1} \cdot \mathrm{h}^{-1}$ intravenous continuous infusion). Control animals $(n=6)$ received crystalloid solution. Global and regional myocardial functions were analyzed by the left ventricular $+\mathrm{dP} / \mathrm{dt}$ and the percentage segment shortening, respectively. Left ventricular infarct size was measured by tetrazolium staining. Tissue myeloperoxidase activity was measured. Myocardial sections were immunohistochemically stained for nitrotyrosine. Coronary microvessel function was studied by videomicroscopy.

Results: Myocardial infarct size was decreased with aprotinin treatment $(27.0 \% \pm$ $3.5 \%$ vs $45.3 \% \pm 3.0 \%$, aprotinin vs control; $P<.05$ ). Myocardium from the ischemic territory showed diminished nitrotyrosine staining in aprotinin-treated animals versus controls, and this was significant by grade $(1.3 \pm 0.2$ vs $3.2 \pm 0.2$, aprotinin vs control; $P<.01)$. In the aprotinin group, coronary microvessel relaxation improved most in response to the endothelium-dependent agonist adenosine diphosphate $(44.7 \% \pm 3.2 \%$ vs $19.7 \% \pm 1.7 \%$, aprotinin vs control; $P<.01)$. No significant improvements in myocardial function were observed with aprotinin treatment.

Conclusions: Aprotinin reduces reperfusion injury after regional ischemia and cardioplegic arrest. Protease inhibition may represent a molecular strategy to prevent postoperative myocardial injury after surgical revascularization with cardiopulmonary bypass.

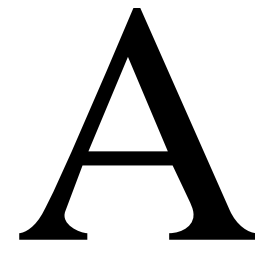

s percutaneous coronary interventions have become standard treatment options for multivessel coronary artery disease, patients referred for coronary artery bypass grafting have become a higher-risk group with more severe coronary disease and preoperative myocardial dysfunction. Coronary bypass grafts deliver blood flow to ischemic areas of the myocardium but also result in regional reperfusion injury, and the use of cardiopulmonary bypass $(\mathrm{CPB})$ with 
cardioplegic arrest introduces a mechanism of further myocardial injury caused by global reperfusion. Reperfusion injury is associated with myocardial stunning, ${ }^{1,2}$ coronary microvascular dysfunction, ${ }^{3,4}$ and myocardial damage and necrosis. ${ }^{5}$ Cardiac dysfunction due to reperfusion injury increases the risk of postoperative complications because of impaired organ perfusion despite successful myocardial revascularization. The serine protease inhibitor aprotinin has been shown to attenuate the inflammatory response to $\mathrm{CPB}^{6}$ and myocardial reperfusion injury. ${ }^{7}$ Specifically, aprotinin has been suggested to reduce reperfusion injury in animal models of regional ischemia, ${ }^{8-10}$ isolated heart models of cardioplegic arrest, ${ }^{11,12}$ and animal models of cardioplegic arrest. ${ }^{11,13}$ Although these studies have described favorable effects of aprotinin in these experimental models, a clinically relevant model that examines both regional ischemia and $\mathrm{CPB}$ with cardioplegic arrest and that simulates surgical revascularization in patients has not been used to evaluate aprotinin therapy. In addition, the role of aprotinin in preventing coronary microvascular dysfunction due to reperfusion injury after regional ischemia and CPB has not been studied. We hypothesized that aprotinin treatment would reduce reperfusion injury in a model of regional ischemia, cardioplegic arrest, and reperfusion. We propose that, in addition to improvements in myocardial protection and reductions in markers of inflammation and tissue injury, aprotinin also prevents dysfunction of the coronary microcirculation, a critical determinant of myocardial perfusion.

\section{Methods \\ Animals}

Animals were housed individually and provided with laboratory chow and water ad libitum. All experiments were approved by the Beth Israel Deaconess Medical Center Animal Care and Use Committee and the Harvard Medical Area Standing Committee on Animals (Institutional Animal Care and Use Committee) and conformed to the US National Institutes of Health guidelines regulating the care and use of laboratory animals (National Institutes of Health publication 5377-3; 1996).

\section{Experimental Design}

Pigs (30 to $35 \mathrm{~kg}$ ) were divided randomly into control $(\mathrm{n}=6)$ and aprotinin treatment $(n=6)$ groups. Groups were subjected to regional left ventricular (LV) ischemia by left anterior descending coronary artery (LAD) occlusion distal to the first diagonal branch for 30 minutes before CPB. Animals underwent CPB with cardioplegic arrest for 60 minutes. After 5 minutes of CPB, a period of 45 minutes of hyperkalemic cardioplegic arrest followed. The aortic crossclamp then was removed and the LAD occlusion released to reperfuse the myocardium for 10 minutes on $\mathrm{CPB}$, after which the animal was weaned from CPB. The myocardium was reperfused for a total of 90 minutes after CPB. The treatment group received aprotinin systemically (Trasylol; aprotinin injection; Bayer Pharmaceuticals Corporation, West Haven, Conn) as follows: a 40,000 kallikrein-inhibiting unit (KIU)/kg intravenous (IV) loading dose, a 40,000 KIU/kg CPB circuit prime, and a 10,000 $\mathrm{KIU} \cdot \mathrm{kg}^{-1} \cdot \mathrm{h}^{-1} \mathrm{IV}$ continuous infusion. The control group was administered IV crystalloid solution. Arterial blood gas (ABG), arterial blood pressure, hematocrit, LV pressure, coronary blood flow, heart rate, electrocardiogram, oxygen saturation, and temperature were monitored.

\section{Surgical Procedure}

Pigs were anesthetized with intramuscular ketamine hydrochloride $(20 \mathrm{mg} / \mathrm{kg})$ and xylazine $(15 \mathrm{mg} / \mathrm{kg})$. General anesthesia with isoflurane gas was maintained by endotracheal intubation and mechanical ventilation, which was held during CPB. The right internal jugular vein and carotid artery were cannulated for monitoring. Through a median sternotomy, a catheter-tipped manometer was placed through the LV apex for pressure measurement. Four 2-mm ultrasonic crystals (Sonometrics Corporation, Ontario, Canada) were placed in the subepicardial layer of the distal LAD territory for analysis of regional myocardial function. A 2-mm ultrasonic coronary flowprobe (Transonic Systems Inc, Ithaca, NY) was placed distal to the site of LAD occlusion. Pigs were given IV heparin $(300 \mathrm{U} / \mathrm{kg})$ and cannulated via the distal ascending aorta and right atrium. A vessel loop was passed around the LAD distal to the first diagonal branch for occlusion. CPB was initiated with a kaolin-activated clotting time of more than 480 seconds, which was maintained with repeat administrations of IV heparin. The proximal aorta was crossclamped, and cold crystalloid cardioplegia was infused into the aortic root. An initial 300 $\mathrm{mL}$ of cold high-potassium cardioplegia $\left(0^{\circ} \mathrm{C}\right.$ to $4{ }^{\circ} \mathrm{C} ; \mathrm{K}^{+} 25$ $\mathrm{mmol} / \mathrm{L})$ was administered, followed by $150 \mathrm{~mL}$ of cold lowpotassium cardioplegia $\left(0^{\circ} \mathrm{C}\right.$ to $\left.4^{\circ} \mathrm{C} ; \mathrm{K}^{+} 12 \mathrm{mmol} / \mathrm{L}\right)$, every 15 minutes. The composition of the crystalloid cardioplegic solution was $(\mathrm{mmol} / \mathrm{L}) \mathrm{NaCl} 121, \mathrm{KCl} 25$ or $12, \mathrm{NaHCO}_{3} 12$, and glucose 11.

\section{Measurement of Global and Regional Myocardial Function}

Global myocardial function was assessed by calculating the maximum positive first derivative of LV pressure over time $(+\mathrm{dP} / \mathrm{dt})$. Regional myocardial function was determined by using subepicardial 2-mm ultrasonic probes (Sonometrics Corporation) to calculate the percentage segment shortening (\%SS), which was normalized to the baseline.

\section{Coronary Microvessel Studies}

Coronary arterioles (60-180 $\mu \mathrm{m}$ internal diameter) were dissected from the LV tissue of the ischemic distal LAD-dependent region. Microvessel studies were performed by in vitro organ bath videomicroscopy as previously described. ${ }^{14}$ Endothelium-dependent relaxation to adenosine diphosphate $\left(10^{-9}\right.$ to $\left.10^{-4} \mathrm{~mol} / \mathrm{L}\right)$ and substance $\mathrm{P}\left(10^{-14}\right.$ to $\left.10^{-6} \mathrm{~mol} / \mathrm{L}\right)$ and endothelium-independent relaxation responses to sodium nitroprusside (SNP; $10^{-9}$ to $10^{-4}$ $\mathrm{mol} / \mathrm{L})$ were examined.

\section{Myocardial Infarct Size, Nitrotyrosine Staining, and Myeloperoxidase Activity}

At the completion of 90 minutes of post-CPB reperfusion, the LV ischemic area and infarct size were measured by triphenyl tetrazolium chloride staining as previously described. ${ }^{15}$ Nitrotyrosine 

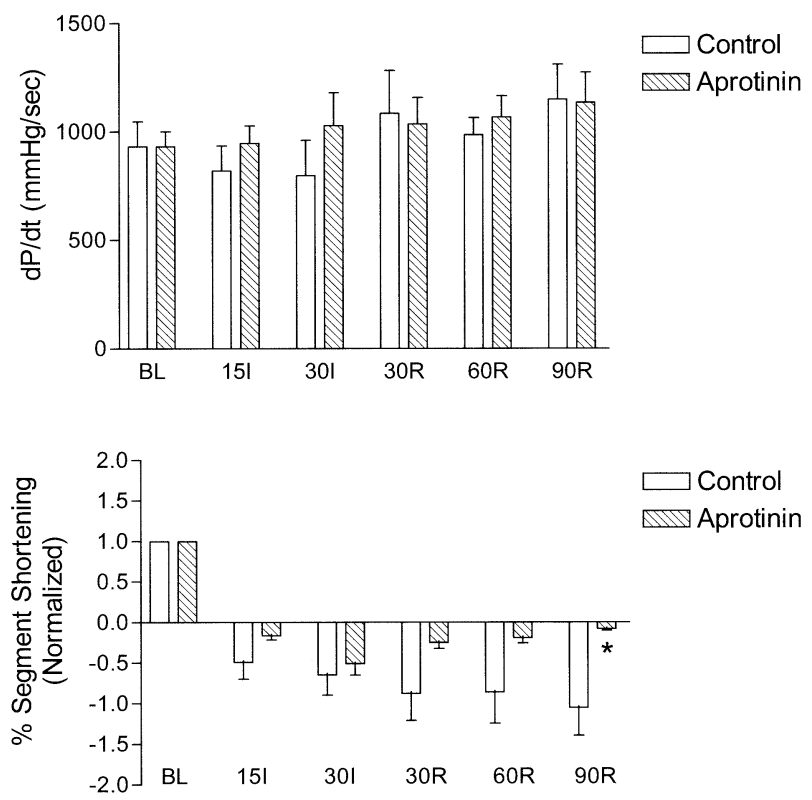

Figure 1. Global and regional myocardial function. The $L V+d P / d t$ was similar in both the control and treatment groups at baseline, during regional ischemia, and in the period of post-CPB reperfusion (top). Both groups showed dyskinesis of the distal LAD territory, as seen by negative percentage segment shortening during regional ischemia and post-CPB reperfusion. Whereas the impairment in regional function was characterized by increasing dyskinesis in the control group, aprotinin treatment resulted in a trend of decreasing dyskinesis, with a significant difference at 90 minutes of post-CPB reperfusion ( ${ }^{*} P<.05$ vs control). $B L$, Baseline; 15I, 15 minutes of ischemia; 30l, 30 minutes of ischemia; 30R, 30 minutes of reperfusion; $60 R, 60$ minutes of reperfusion; $90 R, 90$ minutes of reperfusion.

staining as a measure of peroxynitrite was performed on myocardial tissue from the distal LAD territory by immunohistochemistry as previously described. ${ }^{16}$ All immunohistochemical samples were graded ( 0 to $4 ; 0$ indicated no staining, and 4 indicated dark staining) by a blinded investigator. Myocardial tissue from the distal LAD territory was harvested, and myeloperoxidase (MPO) activity was measured as previously described. ${ }^{17}$ Assessment of this assay in our laboratory demonstrated a linear relationship $(r=$ 0.92 ) such that $1 \mathrm{U}$ of MPO activity correlated with $2.9 \times 10^{6}$ porcine neutrophils.

\section{Statistical Analysis}

Data are shown as mean \pm SEM. Statistical analyses were performed with the Mann-Whitney $U$ test and analysis of variance as appropriate.

\section{Results}

Hemodynamic Parameters, ABG, Temperature, and Hematocrit

No significant differences in heart rate, mean arterial blood pressure, ABG parameters, or temperature were observed

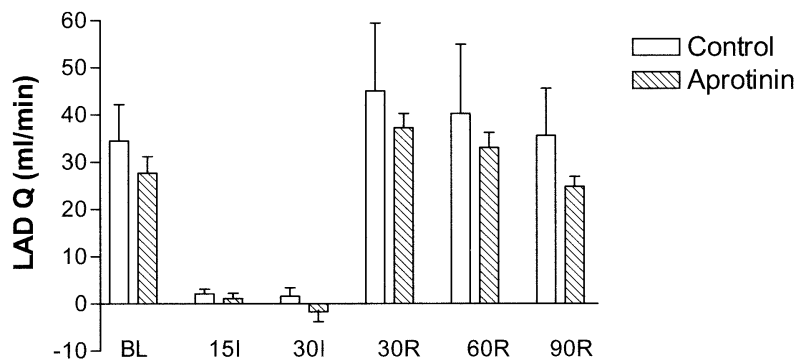

Figure 2. Coronary blood flow. No significant differences were observed in coronary blood flow between groups. $B L$, Baseline; 15I, 15 minutes of ischemia; 30I, 30 minutes of ischemia; 30R, 30 minutes of reperfusion; 60R, 60 minutes of reperfusion; $90 R, 90$ minutes of reperfusion.

between groups. Hematocrit was similar in the control $(21.4 \% \pm 0.7 \%)$ and aprotinin $(22.6 \% \pm 1.0 \%)$ groups.

\section{Global and Regional Myocardial Function and} Coronary Blood Flow

No significant differences in the $\mathrm{LV}+\mathrm{dP} / \mathrm{dt}$ were observed between groups (Figure 1, top). In control and aprotinintreated animals, regional ischemia resulted in dyskinesis in the distal LAD territory, resulting in negative \%SS (Figure 1 , bottom). During post-CPB reperfusion, the regional myocardial function showed trends of increasing dyskinesis in the control group and decreasing dyskinesis in the aprotinin group, with a significant difference at 90 minutes of postCPB reperfusion $(-0.08 \% \pm 0.02 \%$ vs $-1.05 \% \pm 0.3 \%$ $\mathrm{SS}$, aprotinin vs control, respectively; $P<.05)$. No significant differences were observed between groups in coronary blood flow (Figure 2).

Myocardial Infarct Size, Nitrotyrosine Staining, and MPO Activity

The myocardial infarct size, measured as a percentage of the ischemic area, was significantly reduced in the aprotinin group compared with controls $(27.0 \% \pm 3.5 \%$ vs $45.3 \% \pm$ $3.0 \%$, aprotinin vs control, respectively; $P<.05$; Figures 3 and 4). Myocardial sections stained for nitrotyrosine as a measure of peroxynitrite showed reduced staining by grade in the aprotinin group compared with controls $(1.3 \pm 0.2 \mathrm{vs}$ $3.2 \pm 0.2$, aprotinin vs control, respectively; $P<.01$; Figure 5). MPO activity as a measure of neutrophil infiltration was decreased in myocardial tissue from aprotinintreated animals compared with control animals $(0.09 \pm 0.03$ $\mathrm{U} / \mathrm{mg}$ of tissue vs $0.26 \pm 0.07 \mathrm{U} / \mathrm{mg}$ of tissue, aprotinin vs control, respectively; $P<.05$; Figure 6).

\section{Coronary Microvascular Function}

Aprotinin treatment produced increased relaxation responses of coronary arterioles to the endothelium-dependent agonists adenosine diphosphate and substance P compared 
with controls (Figure 7, top and middle, respectively). The relaxation responses to the endothelium-independent agonist SNP were also increased in the aprotinin group compared with controls (Figure 7, bottom).

\section{Discussion}

The principal findings of the study are that in a porcine model of regional myocardial ischemia and cardioplegic arrest followed by reperfusion, aprotinin treatment has the following effects: (1) reduction of myocardial infarct size, (2) prevention of neutrophil accumulation and peroxynitrite generation, and (3) enhancement of coronary microvascular relaxation. An important aspect of this study is that the beneficial effects of aprotinin were observed after both regional ischemia of the LAD territory and global ischemia with cardioplegic arrest. Our results in this clinically relevant model suggest that patients undergoing coronary artery bypass grafting with $\mathrm{CPB}$ and cardioplegic arrest for unstable angina may similarly benefit from an adjunctive therapy of serine protease inhibition to prevent myocardial reperfusion injury.

In our study, aprotinin treatment produced a reduction in myocardial infarct size as less of the ischemic LAD territory progressed to infarction. A previous study of coronary occlusion in a dog model showed decreased myocardial necrosis with aprotinin, ${ }^{18}$ yet another study of regional myocardial ischemia in sheep suggested that aprotinin therapy resulted in increased myocardial damage. ${ }^{19}$ Although these results from animal models are inconsistent, several other studies have provided evidence that aprotinin reduces cardiac enzyme release due to reperfusion injury. Both in a rat model of regional ischemia and an isolated, perfused heart model of cardioplegic arrest, aprotinin treatment resulted in a decreased release of creatine kinase. ${ }^{10,12}$ Furthermore, in patients, aprotinin therapy has been associated with lower levels of cardiac troponins after cardiac surgery. ${ }^{20,21}$

Neutrophils play a critical role in reperfusion injury through a process of adhering to the vascular endothelium, transmigrating, and accumulating in the reperfused tissue. During this course, neutrophils are activated and release cytotoxic metabolites, proteolytic enzymes, and cytokines, leading to tissue injury and to recruitment of more neutrophils. In this study, in the aprotinin group, we observed an attenuation of neutrophil infiltration by decreased MPO activity in myocardial tissue from the reperfused LAD territory. Aprotinin has been shown to reduce neutrophil extravasation indirectly by measuring MPO activity ${ }^{10}$ and directly through intravital microscopy. ${ }^{22}$ Furthermore, we observed that aprotinin treatment resulted in decreased nitrotyrosine staining of myocardial tissue sections from the ischemic LAD territory. Tissue nitrotyrosine is a metabolic product and an indirect measure of peroxynitrite, a nitrogen radical that is produced during reperfusion injury along with

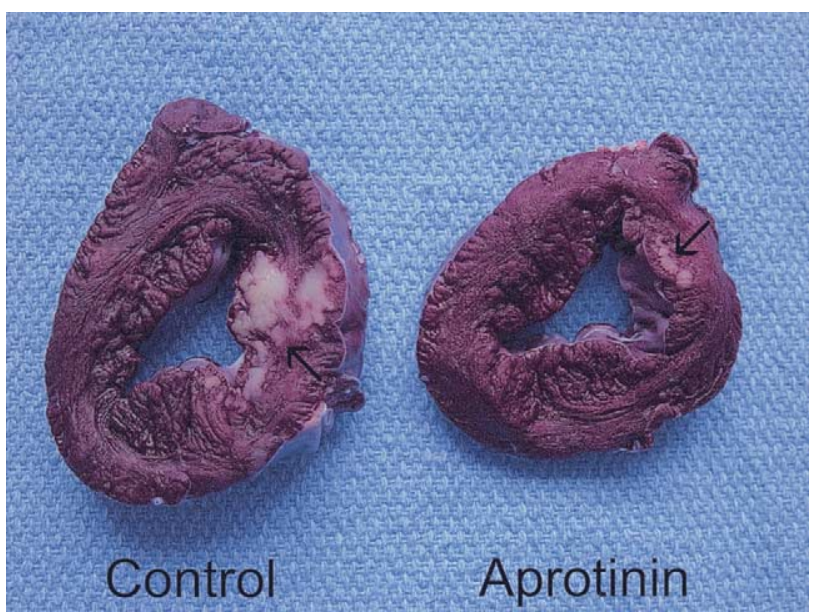

Figure 3. Representative image of LV infarct size. Triphenyl tetrazolium chloride staining was used to determine the LV infarct size (arrows), which was markedly decreased in the LV myocardium of aprotinin-treated animals (right) compared with controls (left).

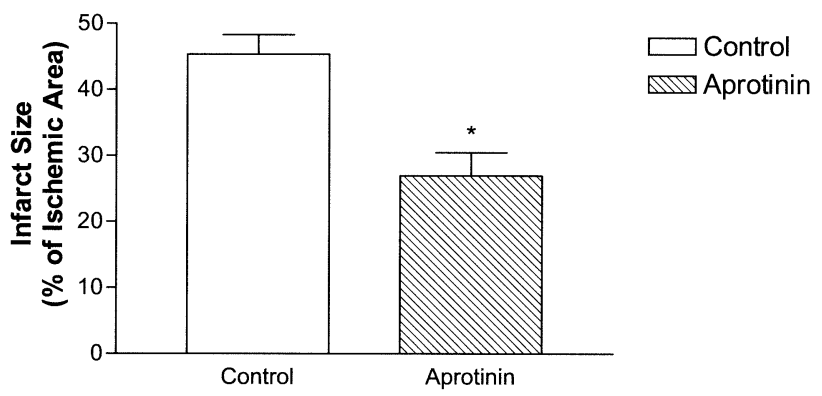

Figure 4. Quantification of LV infarct size. Infarct size was measured as a percentage of the $L V$ ischemic area. The percent of the LV ischemic area that progressed to infarction was significantly reduced in the aprotinin group compared with the LV myocardium from control animals ( $\left.{ }^{*} P<.05\right)$.

oxygen radicals. Peroxynitrite is produced in patients during cardiac operations ${ }^{23}$ and has been suggested to contribute to reperfusion injury. In a previous study from our laboratory, treatment with FP-15, a peroxynitrite inhibitor, decreased the extent of reperfusion injury in a pig model of regional myocardial ischemia. ${ }^{16}$ In addition to reducing nitrogen radical production, aprotinin has been shown to prevent oxygen radical formation. ${ }^{24}$ On the basis of these observations, mechanisms by which aprotinin attenuates reperfusion injury likely include prevention of neutrophil extravasation and production of oxygen and nitrogen radicals.

Finally, aprotinin therapy was associated with improved coronary microvascular relaxation, which was impaired because of reperfusion injury. ${ }^{3,4}$ In our study, aprotinin enhanced relaxation responses to both endothelium-dependent 


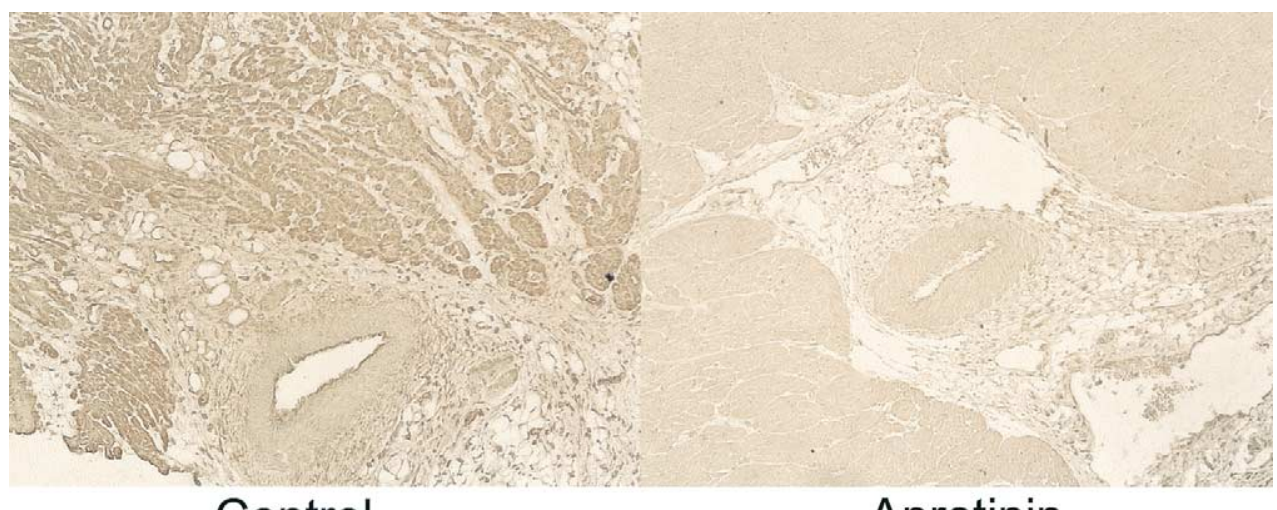

Control

Aprotinin

Figure 5. Myocardial nitrotyrosine staining. Aprotinin treatment decreased staining for nitrotyrosine, a measure of tissue peroxynitrite, as shown in the representative myocardial sections from the distal LAD territory of aprotinintreated (right) and control (left) animals.

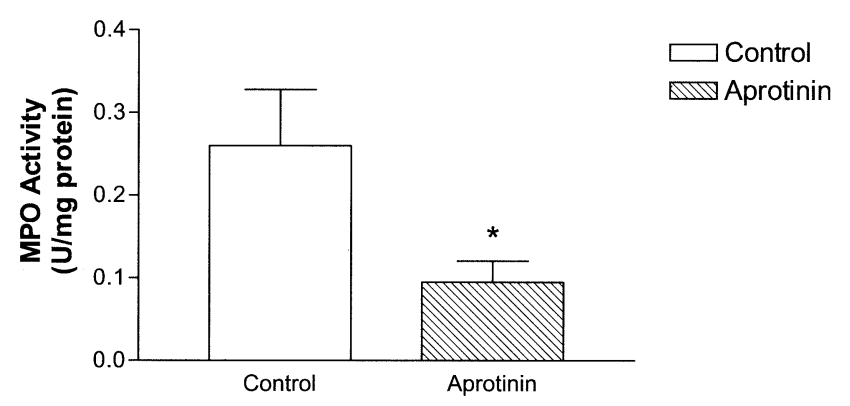

Figure 6. Tissue MPO activity. Tissue MPO activity was determined as a measure of neutrophil infiltration. Myocardium from the distal LAD territory had reduced MPO activity with aprotinin therapy compared with controls $\left({ }^{*} P<.05\right)$.

(adenosine diphosphate and substance P) and endotheliumindependent (SNP) agonists. Although evidence suggests that aprotinin increases endothelium-dependent relaxation to acetylcholine after hypothermic circulatory arrest, ${ }^{25}$ the results of our study did not differentiate between endothelium-dependent and endothelium-independent mechanisms of coronary vascular smooth muscle relaxation. The mechanism by which aprotinin improves coronary microvascular relaxation after reperfusion injury likely involves the attenuation of neutrophil activity. ${ }^{26}$

A limitation of the study is that crystalloid cardioplegia was used. Blood cardioplegia has been associated with greater myocardial protection in laboratory experiments, ${ }^{27,28}$ and clinical studies have suggested that blood cardioplegia, compared with crystalloid cardioplegia, may improve outcomes in patients with severe preoperative myocardial dysfunction. ${ }^{29,30}$ Although blood cardioplegia was not used in our study, there is evidence that aprotinin prevents myocardial injury, as measured by troponin re- lease, similarly in patients who undergo cardioplegic arrest with blood or crystalloid solutions. ${ }^{21}$ Another limitation of the study is that although aprotinin treatment reduced myocardial infarction and enhanced coronary microvascular relaxation, improvement in global and regional myocardial function was not shown. Although during post-CPB reperfusion, aprotinin treatment attenuated the dyskinesis in the reperfused LAD territory, the resulting akinesis would be unlikely to provide a significant improvement in regional myocardial function. A possible explanation for these results is that after 30 minutes of regional ischemia and 45 minutes of cardioplegic arrest, the stunned myocardium was still in the recovery phase, which may require 48 hours or longer after such periods of ischemia for significant restoration of myocardial function. ${ }^{1,2}$ Overall, although no functional improvement was shown with aprotinin treatment, the trend of a progressive decrease in dyskinesis during the post-CPB reperfusion period suggests that aprotinin reduces the deleterious effects of reperfusion injury on regional LV function.

In conclusion, the results of our study show that aprotinin reduces reperfusion injury in a pig model of regional ischemia and cardioplegic arrest, as seen by decreased infarct size, prevention of neutrophil tissue infiltration and nitrogen radical production, and increased coronary microvascular relaxation. Although the use of aprotinin may not translate into a clinically significant benefit in routine patients who are surgically revascularized, high-risk patients—such as those with severe coronary artery disease, preoperative cardiac dysfunction, and diabetes mellitus-may be the population that will have greater potential gain from the cardioprotective effects of serine protease inhibition. As the current trend of aggressive coronary artery disease management by percutaneous coronary intervention results in increasingly high-risk patients referred for cardiac surgery, 

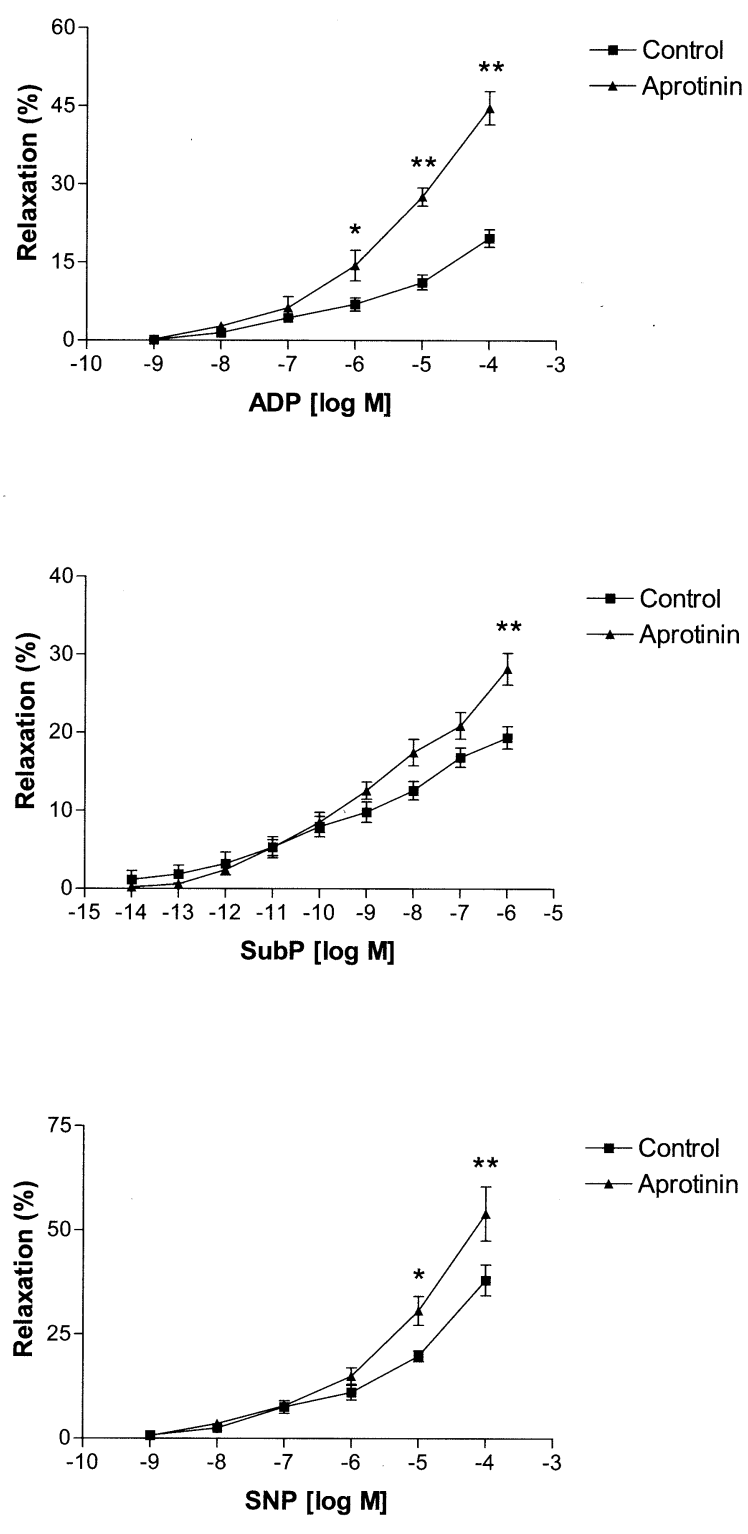

Figure 7. Coronary microvascular relaxation. Endothelium-dependent coronary microvessel relaxation responses were markedly increased with adenosine diphosphate (ADP) and also with substance $P(S u b P)$ with aprotinin treatment compared with controls. Aprotinin also increased coronary microvessel relaxation in response to the endothelium-independent vasodilator sodium nitroprusside (SNP); ${ }^{*} P<.05$ and ${ }^{* *} P<.01$ vs control).

therapy such as serine protease inhibition with aprotinin may allow for continued successful outcomes as cardiac surgeons are presented with more challenging cases for coronary revascularization.

\section{References}

1. Kloner RA, Jennings RB. Consequences of brief ischemia: stunning, preconditioning, and their clinical implications-part 1. Circulation. 2001;104:2981-9.
2. Kloner RA, Jennings RB. Consequences of brief ischemia: stunning, preconditioning, and their clinical implications-part 2. Circulation. 2001;104:3158-67.

3. Sellke FW, Boyle EM Jr, Verrier ED. Endothelial cell injury in cardiovascular surgery: the pathophysiology of vasomotor dysfunction. Ann Thorac Surg. 1996;62:1222-8.

4. Park JL, Lucchesi BR. Mechanisms of myocardial reperfusion injury. Ann Thorac Surg. 1999;68:1905-12.

5. Ambrosio G, Tritto I. Reperfusion injury: experimental evidence and clinical implications. Am Heart J. 1999;138:S69-75.

6. Mojcik CF, Levy JH. Aprotinin and the systemic inflammatory response after cardiopulmonary bypass. Ann Thorac Surg. 2001;71:74554.

7. Bull DA, Maurer J. Aprotinin and preservation of myocardial function after ischemia-reperfusion injury. Ann Thorac Surg. 2003;75:S735-9.

8. McCarthy RJ, Tuman KJ, O'Connor C, Ivankovich AD. Aprotinin pretreatment diminishes postischemic myocardial contractile dysfunction in dogs. Anesth Analg. 1999;89:1096-100.

9. Hendrikx M, Rega F, Jamaer L, Valkenborgh T, Gutermann H, Mees U. $\mathrm{Na}(+) / \mathrm{H}(+)$-exchange inhibition and aprotinin administration: promising tools for myocardial protection during minimally invasive CABG. Eur J Cardiothorac Surg. 2001;19:633-9.

10. Pruefer D, Buerke U, Khalil M, Dahm M, Darius H, Oelert H, et al. Cardioprotective effects of the serine protease inhibitor aprotinin after regional ischemia and reperfusion on the beating heart. $J$ Thorac Cardiovasc Surg. 2002;124:942-9.

11. Sunamori M, Sultan I, Suzuki A. Effect of aprotinin to improve myocardial viability in myocardial preservation followed by reperfusion. Ann Thorac Surg. 1991;52:971-8.

12. Gurevitch J, Barak J, Hochhauser E, Paz Y, Yakirevich V. Aprotinin improves myocardial recovery after ischemia and reperfusion. Effects of the drug on isolated rat hearts. J Thorac Cardiovasc Surg. 1994; 108:109-18.

13. Sunamori M, Innami R, Amano J, Suzuki A, Harrison CE. Role of protease inhibition in myocardial preservation in prolonged hypothermic cardioplegia followed by reperfusion. Effect of aprotinin in an experimental model. J Thorac Cardiovasc Surg. 1988;96:314-20.

14. Khan TA, Ruel M, Bianchi C, Voisine P, Komjati K, Szabo C, et al. Poly(ADP-ribose) polymerase inhibition improves postischemic myocardial function after cardioplegia-cardiopulmonary bypass. J Am Coll Surg. 2003;197:270-7.

15. Uematsu M, Gaudette GR, Laurikka JO, Levitsky S, McCully JD. Adenosine-enhanced ischemic preconditioning decreases infarct in the regional ischemic sheep heart. Ann Thorac Surg. 1998;66:382-7.

16. Bianchi C, Wakiyama H, Faro R, Khan T, McCully JD, Levitsky S, et al. A novel peroxynitrite decomposer catalyst (FP-15) reduces myocardial infarct size in an in vivo peroxynitrite decomposer and acute ischemia-reperfusion in pigs. Ann Thorac Surg. 2002;74:1201-7.

17. Amsterdam EA, Stahl GL, Pan HL, Rendig SV, Fletcher MP, Longhurst JC. Limitation of reperfusion injury by a monoclonal antibody to C5a during myocardial infarction in pigs. Am J Physiol. 1995;268: H448-57.

18. Diaz PE, Fishbein MC, Davis MA, Askenazi J, Maroko PR. Effect of the kallikrein inhibitor aprotinin on myocardial ischemic injury after coronary occlusion in the dog. Am J Cardiol. 1977;40:541-9.

19. Bukhari EA, Krukenkamp IB, Burns PG, Gaudette GR, Schulman JJ, al-Fagih MR, et al. Does aprotinin increase the myocardial damage in the setting of ischemia and preconditioning? Ann Thorac Surg. 1995; 60:307-10.

20. Wendel HP, Heller W, Michel J, Mayer G, Ochsenfahrt C, Graeter U, et al. Lower cardiac troponin $\mathrm{T}$ levels in patients undergoing cardiopulmonary bypass and receiving high-dose aprotinin therapy indicate reduction of perioperative myocardial damage. $J$ Thorac Cardiovasc Surg. 1995;109:1164-72.

21. Hendrikx M, Jiang H, Gutermann H, Toelsie J, Renard D, Briers A, et al. Release of cardiac troponin I in antegrade crystalloid versus cold blood cardioplegia. J Thorac Cardiovasc Surg. 1999;118:452-9.

22. Asimakopoulos G, Thompson R, Nourshargh S, Lidington EA, Mason JC, Ratnatunga CP, et al. An anti-inflammatory property of aprotinin detected at the level of leukocyte extravasation. J Thorac Cardiovasc Surg. 2000;120:361-9. 
23. Hayashi Y, Sawa Y, Ohtake S, Fukuyama N, Nakazawa H, Matsuda H. Peroxynitrite formation from human myocardium after ischemiareperfusion during open heart operation. Ann Thorac Surg. 2001;72: 571-6.

24. Hallett MB, Shandall A, Young HL. Mechanism of protection against "reperfusion injury" by aprotinin. Roles of polymorphonuclear leucocytes and oxygen radicals. Biochem Pharmacol. 1985;34:1757-61.

25. Aoki M, Jonas RA, Nomura F, Stromski ME, Tsuji MK, Hickey PR, et al. Effects of aprotinin on acute recovery of cerebral metabolism in piglets after hypothermic circulatory arrest. Ann Thorac Surg. 1994; 58:146-53

26. Kadletz M, Dignan RJ, Loesser KE, Hess ML, Wechsler AS. Ischemia and activated neutrophils alter coronary microvascular but not epicardial coronary artery reactivity. J Thorac Cardiovasc Surg. 1994;108: 648-57.
27. Vinten-Johansen J, Edgerton TA, Hansen KJ, Carroll P, Mills SA, Cordell AR. Surgical revascularization of acute (1 hour) coronary occlusion: blood versus crystalloid cardioplegia. Ann Thorac Surg. 1986;42:247-54.

28. Bolling K, Kronon M, Allen BS, Wang T, Ramon S, Feinberg H. Myocardial protection in normal and hypoxically stressed neonatal hearts: the superiority of blood versus crystalloid cardioplegia. J Thorac Cardiovasc Surg. 1997;113:994-1003; discussion 1003-5.

29. Ibrahim MF, Venn GE, Young CP, Chambers DJ. A clinical comparative study between crystalloid and blood-based St Thomas' hospital cardioplegic solution. Eur J Cardiothorac Surg. 1999;15:75-83.

30. Flack JE III, Cook JR, May SJ, Lemeshow S, Engelman RM, Rousou $\mathrm{JA}$, et al. Does cardioplegia type affect outcome and survival in patients with advanced left ventricular dysfunction? Results from the CABG Patch Trial. Circulation. 2000;102:III84-9. 